\title{
STABILITY AND GLOBAL ATTRACTIVITY FOR A CLASS OF NONLINEAR DELAY DIFFERENCE EQUATIONS
}

\author{
BINXIANG DAI AND NA ZHANG
}

Received 13 April 2005

A class of nonlinear delay difference equations are considered and some sufficient conditions for global attractivity of solutions of the equation are obtained. It is shown that the stability properties, both local and global, of the equilibrium of the delay equation can be derived from those of an associated nondelay equation.

\section{Introduction}

Consider the following nonlinear delay difference equations

$$
x(n+1)=c x(n)+f(x(n)-x(n-k))
$$

where $c \in[0,1)$ is a given constant, $k$ is a positive integer, $f: R \rightarrow R$ is continuous and $f(0)=0, f(u) \neq 0$ for $u \neq 0$. Such a equation arises from some of the earliest mathematical models of the macroeconomic "trade cycle," and have attracted a great deal of attention (see, e.g., $[1,4,5,6,7,8,9,10]$ and references cited therein). When $k=1$, Sedaghat [9] obtained some sufficient conditions for the permanence and boundedness by exploring the relationship between the first order equations and the higher order equations.

Our main goal in this paper is to obtain some sufficient conditions which guarantee that the equilibrium of (1.1) is a global attractor. We still investigate the stability of (1.1) and show that the stability properties, both local and global, of the equilibrium of the delay equation (1.1) can be derived from those of the associated nondelay equation

$$
x(n+1)=f(x(n))
$$

where the $f$ is the same function as in (1.1). This result is of considerable benefit to the study of delay-difference equations of this type since the stability properties of nondelay difference equations are better understood $[2,3]$.

A point $\bar{x}$ is called an equilibrium of $(1.1)$ if $x(n)=\bar{x}(n \geq 0)$ is a solution of (1.1). It is obvious that (1.1) has the only equilibrium $\bar{x}=0$ under the hypothesis. 
We say that the equilibrium $\bar{x}=0$ of (1.1) is a global attractor if and only if, for arbitrary initial conditions, the corresponding solution $x(n)$ of (1.1) satisfies $\lim _{n \rightarrow \infty} x(n)=0$. The region of attraction of the equilibrium $\bar{x}=0$ is defined as the set of all initial points $\{x(-k), x(-k+1), \ldots, x(0)\}$ such that $\lim _{n \rightarrow \infty} x(n)=0$.

Without loss generality, throughout this paper the norm will be defined as

$$
\|x\|=\max _{1 \leq i \leq m}\left|x_{i}\right|, \quad x \in R^{m}
$$

The rest of the paper is organized as follows. In Section 2, we derive a sufficient condition for global attractivity of the equilibrium of (1.1). In Section 3, we discuss the stability properties of (1.1).

\section{Global Attractivity of (1.1)}

The objective of this section is to derive sufficient conditions which guarantee that the equilibrium of (1.1) is a global attractor. Let

$$
u(n)=x(n)-x(n-k) .
$$

Then (1.1) is reduced to:

$$
u(n+1)=c u(n)+f(u(n))-f(u(n-k)) .
$$

Noting that $c \in[0,1),(2.2)$ has the unique equilibrium $\bar{u}=0$. We first show the following proposition.

Proposition 2.1. Assume that there exist a constant $\alpha \in(0,1)$ such that $\alpha+c<1$ and

$$
|f(u)| \leq \alpha|u|
$$

for all $u$. Then every solution $u(n)$ of (2.2) satisfies

$$
\lim _{n \rightarrow \infty} u(n)=0 .
$$

Proof. By (2.2) and the assumption of $f$, we have

$$
\begin{aligned}
|u(n+1)| & =|c u(n)+f(u(n))-f(u(n-k))| \\
& \leq c|u(n)|+\alpha|u(n)|+\alpha|u(n-k)| \\
& =(\alpha+c)|u(n)|+\alpha|u(n-k)|,
\end{aligned}
$$

for $n=0,1, \ldots$ Using induction and noting that $0<\alpha<1$ and $\alpha+c<1$, we have

$$
\lim _{n \rightarrow \infty}|u(n)|=0
$$

which implies that $\lim _{n \rightarrow \infty} u(n)=0$. The proof is complete.

The following theorem gives a sufficient condition for the equilibrium $\bar{x}=0$ of (1.1) to be a global attractor. 
THeOREM 2.2. If the condition (2.3) holds, then every solution of (1.1) converges to $\bar{x}=0$.

Proof. Let

$$
u(n)=x(n)-x(n-k) .
$$

Then (1.1) can be written as

$$
x(n+1)=c x(n)+f(u(n)), \quad \text { for } n=0,1, \ldots
$$

So we have

$$
\begin{aligned}
& x(1)=c x(0)+f(u(0)), \\
& x(2)=c x(1)+f(u(1))=c^{2} x(0)+c f(u(0))+f(u(1)) .
\end{aligned}
$$

By induction, we get

$$
x(n)=c^{n} x(0)+\sum_{i=0}^{n-1} c^{n-1-i} f(u(i)) .
$$

Noting that $c \in[0,1)$, we have

$$
\lim _{n \rightarrow \infty} c^{n} x(0)=0
$$

Let

$$
\tilde{u}(n)=\sum_{i=0}^{n-1} c^{n-1-i} f(u(i)) .
$$

We distinguish two cases to prove

$$
\lim _{n \rightarrow \infty} \tilde{u}(n)=0 .
$$

Case $1\left(\sum_{i=0}^{\infty} f\left(u(i) / c^{i}<\infty\right)\right.$. In this case, it is obvious that $\lim _{n \rightarrow \infty} \tilde{u}(n)=0$ since $\lim _{n \rightarrow \infty} c^{n-1}=0$.

Case $2\left(\sum_{i=0}^{\infty} f\left(u(i) / c^{i}=\infty\right)\right.$. By Stolz Theorem, we have

$$
\begin{aligned}
\lim _{n \rightarrow \infty} \tilde{u}(n) & =\lim _{n \rightarrow \infty} \frac{\sum_{i=0}^{n-1}\left(f(u(i)) / c^{i}\right)}{1 / c^{n-1}} \\
& =\lim _{n \rightarrow \infty} \frac{\sum_{i=0}^{n}\left(f(u(i)) / c^{i}\right)-\sum_{i=0}^{n-1}\left(f(u(i)) / c^{i}\right)}{\left(1 / c^{n}\right)-\left(1 / c^{n-1}\right)} \\
& =\lim _{n \rightarrow \infty} \frac{f(u(n)) / c^{n}}{(1-c) / c^{n}} \\
& =\frac{1}{1-c} \lim _{n \rightarrow \infty} f(u(n)) .
\end{aligned}
$$


Using the continuity of the function $f$ and Proposition 2.1, we have

$$
\lim _{n \rightarrow \infty} f(u(n))=0
$$

Thus

$$
\lim _{n \rightarrow \infty} \tilde{u}(n)=0
$$

Finally (2.11) and (2.13) imply that $\lim _{n \rightarrow \infty} x(n)=0$. The proof is completed.

\section{Stability of (1.1)}

Let $x(n)$ be a solution of (1.1). We defined the vector $y(n) \in R^{k+1}$ as $y(n)=\left(y_{1}(n), \ldots\right.$, $\left.y_{k+1}(n)\right)^{T}$, where

$$
y_{j}(n)=x(n+j-k-1), \quad j=1,2, \ldots, k+1 .
$$

Then the delay equation $(1.1)$ is equivalent to the following $(k+1)$-dimensional system

$$
y(n+1)=g(y(n)), \quad y(n) \in R^{k+1},
$$

where $g(y)=\left(g_{1}(y), g_{2}(y), \ldots, g_{k+1}(y)\right)^{T}$ with

$$
\begin{aligned}
g_{j}(y(n)) & =y_{j+1}(n), \quad j=1,2, \ldots, k, \\
g_{k+1}(y(n)) & =c y_{k+1}(n)+f\left(y_{k+1}(n)-y_{1}(n)\right) .
\end{aligned}
$$

It is obvious that $\bar{y}=0$ is the only equilibrium of the system (3.2).

In this section, we present the main results which relate the stability properties of the delay equation (1.1) to those of the associated nondelay equation

$$
x(n+1)=f(x(n)), \quad n \geq-k .
$$

First we establish a lemma which will be used in proving the main theorem.

Lemma 3.1. Let $y(n)$ be a solution of the system (3.2). Then for $j=1,2, \ldots, k+1$, the following statements are true:

(a)

$$
y_{j}(n)=y_{j+n}(0), \quad 0 \leq n \leq k+1-j
$$

(b)

$$
\begin{aligned}
\left|y_{j}(n)\right| \leq & c^{n+j-k-1}\left|y_{k+1}(0)\right| \\
& +\sum_{i=0}^{n+j-k-2} c^{n+j-k-2-i}\left|f\left(y_{k+1}(i)-y_{1}(i)\right)\right|, \quad n \geq k+2-j .
\end{aligned}
$$


Proof. From (3.3), we have

$$
\begin{aligned}
y_{k+1}(n) & =c y_{k+1}(n-1)+f\left(y_{k+1}(n-1)-y_{1}(n-1)\right) \\
& =c^{n} y_{k+1}(0)+\sum_{i=0}^{n-1} c^{n-1-i} f\left(y_{k+1}(i)-y_{1}(i)\right) .
\end{aligned}
$$

Now let $1 \leq j \leq k+1$. Equation (3.3) also implies

$$
y_{j}(n)=y_{j+1}(n-1)=y_{j+n}(0), \quad \text { for } 0 \leq n \leq k+1-j,
$$

which leads to (a), and

$$
y_{j}(n)=y_{k+1}(n+j-k-1), \quad \text { for } n \geq k+2-j .
$$

Combined with (3.8), this yields, for $n \geq k+2-j$, that

$$
y_{j}(n)=c^{n+j-k-1} y_{k+1}(0)+\sum_{i=0}^{n+j-k-2} c^{n+j-k-2-i} f\left(y_{k+1}(i)-y_{1}(i)\right) .
$$

This leads to the inequality (3.7), and thus, (b) holds. The proof is completed.

Theorem 3.2. Assume $f$ satisfies

$$
|f(x+y)| \leq|f(x)|+|f(y)|
$$

for all $x, y \in R$. If the equilibrium of (3.5) is stable, then the equilibrium of (1.1) is also stable. Proof. It is sufficient to prove the stability of the equilibrium of (3.2) because of the equivalence of (1.1) and (3.2).

Let $\epsilon>0$ be arbitrary. Since the equilibrium of (3.5) is stable, there exists $\delta_{1}>0$ such that $|x(-k)|<\delta_{1}$ implies $|x(n)|<(1-c) \epsilon / 2$ for all $n \geq-k$. Now choose $\delta=$ $\min \left(\delta_{1},(1-c) \epsilon / 2\right)$, Then $\|y(0)\|<\delta$ implies $|x(-k)|<\delta \leq \delta_{1}$ from the definition of $y$ given by (3.1). Hence,

$$
|x(n)|<\frac{(1-c) \epsilon}{2}
$$

for all $n \geq-k$, which implies

$$
|f(x(n))|<\frac{(1-c) \epsilon}{2}
$$

for all $n \geq-k$. Therefore, for all $n \geq 0$, by (3.1)

$$
\left|f\left(y_{k+1}(n)\right)\right|<\frac{(1-c) \epsilon}{2}, \quad\left|f\left(y_{1}(n)\right)\right|<\frac{(1-c) \epsilon}{2} .
$$

Noting that $f$ satisfies

$$
|f(x+y)| \leq|f(x)|+|f(y)|
$$


we get

$$
\left|f\left(y_{k+1}(n)-y_{1}(n)\right)\right|<(1-c) \epsilon
$$

Now $\|y(0)\|<\delta$ implies that $\left|y_{j}(0)\right|<\delta \leq(1-c) \epsilon / 2<\epsilon$ for $1 \leq j \leq k+1$. Hence, from Lemma 3.1(a),

$$
\left|y_{j}(n)\right|=\left|y_{j+n}(0)\right|<\epsilon, \quad \text { for } 0 \leq n \leq k+1-j,
$$

and from Lemma 3.1(b) and (3.17),

$$
\begin{aligned}
\left|y_{j}(n)\right| & <\epsilon c^{n+j-k-1}+(1-c) \epsilon \frac{c^{n+j-k-2}-1 / c}{1-1 / c} \\
& =\epsilon c^{n+j-k-1}+\epsilon(1-c) \frac{1-c^{n+j-k-1}}{1-c} \\
& =\epsilon, \quad \text { for } n \geq k+2-j .
\end{aligned}
$$

Therefore, for arbitrary $\epsilon>0$, there exists $\delta>0$, such that $\|y(0)\|<\delta$ implies $\|y(n)\|<\epsilon$ for $n \geq 0$, so the equilibrium of (3.2) is stable. This completes the proof.

Theorem 3.3. Assume that (3.12) holds. If there exists a constant $m>0$ such that $G(m)=$ $\{x \in R|| x \mid<m\}$ is a subset of attractive region of the equilibrium of (3.2), then $G(m)$ is also contained in the attractive region of the equilibrium of (1.1).

Proof. Let $\epsilon>0$ be arbitrary. Since $G(m)$ is a subset of attractive region of (3.2), there exists $T_{1}(m, \epsilon)$ such that $|x(-k)|<m$ implies $|x(n)|<\epsilon$ for $n \geq T_{1}$.

Assume that $y(0) \in R^{k+1}$ and $\|y(0)\|<m$, then we have $|x(-k)|<m$. So there exists $T_{2}(m,(1-c) \epsilon / 4) \geq T_{1}$ such that $|x(n)|<(1-c) \epsilon / 4$ for all $n \geq T_{2}$, which implies, by (3.1) and (3.12), that

$$
\left|f\left(y_{k+1}(n)-y_{1}(n)\right)\right|<\frac{(1-c) \epsilon}{2}
$$

for all $n \geq T_{2}+k$. Let $1 \leq j \leq k+1$. By Lemma 3.1, we have

$$
\left|y_{j}(n)\right|<m c^{n+j-k-1}+\frac{\epsilon}{2}+\sum_{i=0}^{T_{2}+k-1} c^{n+j-k-2-i}\left|f\left(y_{k+1}(i)-y_{1}(i)\right)\right|
$$

provided $n \geq k+2-j$ which is true for $n \geq k+1$. Now

$$
\begin{aligned}
\left|f\left(y_{k+1}(i)-y_{1}(i)\right)\right| & =|f(x(i)-x(i-k))| \\
& \leq|f(x(i))|+|f(x(i-k))| \\
& =\left|f^{i+k+1}(x(-k))\right|+\left|f^{i+1}(x(-k))\right|,
\end{aligned}
$$


where $f^{j}=\underbrace{f \circ f \circ \cdots \circ f}_{j}$ means the function $f$ composed with itself $j$ times. The continuity of $f$ implies that $f^{j}$ is also continuous, and so there exists $L>0$ such that $\left|f^{i+k+1}(x(-k))\right|<L$ and $\left|f^{i+1}(x(-k))\right|<L$. From (3.21), we obtain for $n \geq T_{2}+2 k$

$$
\begin{aligned}
\left|y_{j}(n)\right| & <m c^{n+j-k-1}+\frac{\epsilon}{2}+2 L \sum_{i=0}^{T_{2}+k-1} c^{n+j-k-2-i} \\
& <\left(m+\frac{2 L}{1-c}\right) c^{n+j-2 k-1-T_{2}}+\frac{\epsilon}{2} .
\end{aligned}
$$

Now choose $T_{3}$ such that

$$
\left(m+\frac{2 L}{1-c}\right) c^{n+j-2 k-1-T_{2}} \leq \frac{\epsilon}{2}
$$

holds for $n \geq T_{3}$, that is

$$
T_{3} \geq T_{2}+2 k+\frac{\ln (\epsilon / 2(m+(2 L /(1-c))))}{\ln c} .
$$

Then $\|y(0)\|<m$ implies $\|y(n)\|<\epsilon$ for $n \geq T_{3}$. So $G(m)$ is also s subset of attractive region of the equilibrium of (1.1). This completes the proof.

Theorems 3.2 and 3.3 can be combined to give the following corollaries.

Corollary 3.4. Assume that the condition (3.12) holds. If the equilibrium of (3.5) is asymptotically stable, then the equilibrium of (1.1) is also asymptotically stable.

Corollary 3.5. Assume that the condition (3.12) holds. If the equilibrium of (3.5) is globally stable, then the equilibrium of (1.1) is also globally stable.

\section{Acknowledgments}

The work was supported by Hunan Province Natural Science Foundation(02JJY2012) and Natural Science Foundation of Central South University.

\section{References}

[1] J. M. Blatt, Dynamic Economic Systems: A Post-Keynesian Approach, M. E. Sharpe, New York, 1983.

[2] S. N. Elaydi, An Introduction to Difference Equations, Undergraduate Texts in Mathematics, Springer, New York, 1996.

[3] M. E. Fisher, Stability of a class of delay-difference equations, Nonlinear Anal. 8 (1984), no. 6, 645-654.

[4] J. R. Hicks, A Contribution to the Theory of the Trade Cycle, Clarendon Press, Oxford, 1965.

[5] V. L. Kocić and G. Ladas, Global Behavior of Nonlinear Difference Equations of Higher Order with Applications, Mathematics and Its Applications, vol. 256, Kluwer Academic, Dordrecht, 1993.

[6] G. Ladas, Open problems and conjectures, J. Differ. Equations Appl. 8 (2002), 667-671.

[7] G. Papaschinopoulos and C. J. Schinas, Stability of a class of nonlinear difference equations, J. Math. Anal. Appl. 230 (1999), no. 1, 211-222. 


\section{Stability and global attractivity for DDE}

[8] P. A. Samuelson, Interaction between the multiplier analysis and the principle of acceleration, Rev. Econom. Statist. 21 (1939), no. 2, 75-78.

[9] H. Sedaghat, A class of nonlinear second order difference equations from macroeconomics, Nonlinear Anal. 29 (1997), no. 5, 593-603.

[10] Bounded oscillations in the Hicks business cycle model and other delay equations, J. Differ. Equations Appl. 4 (1998), no. 4, 325-341.

Binxiang Dai: School of Mathematical Science and Computing Technology, Central South University, Changsha, Hunan 410075, China

E-mail address: bxdai@hnu.cn

$\mathrm{Na}$ Zhang: College of Mathematics and Econometrics, Hunan University, Changsha, Hunan 410082, China

E-mail address: zn19791020@tom.com 


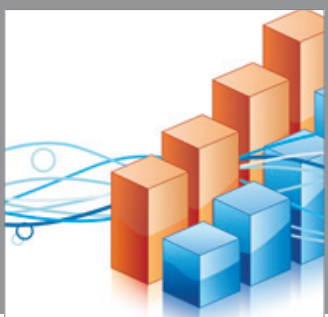

Advances in

Operations Research

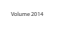

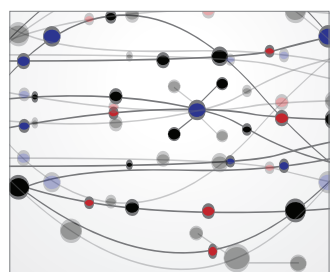

\section{The Scientific} World Journal
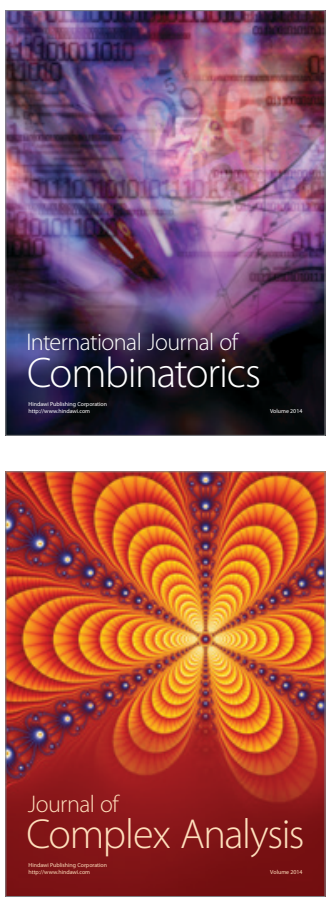

International Journal of

Mathematics and

Mathematical

Sciences
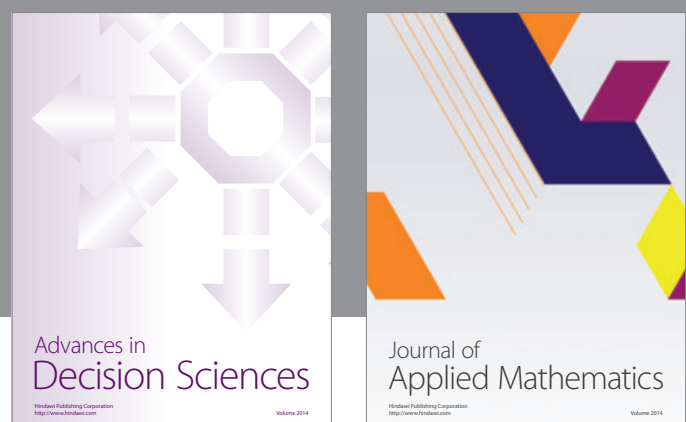

Journal of

Applied Mathematics
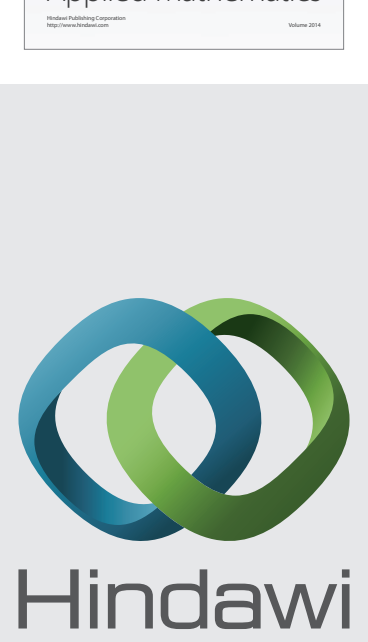

Submit your manuscripts at http://www.hindawi.com
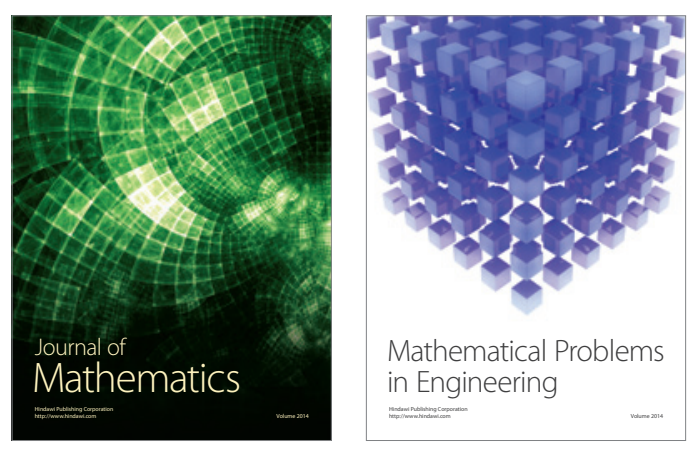

Mathematical Problems in Engineering
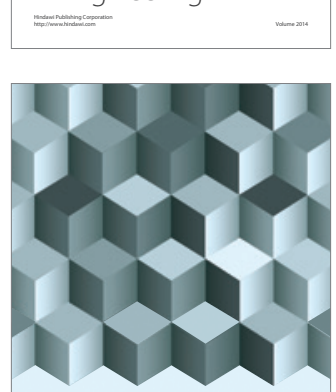

Journal of

Function Spaces
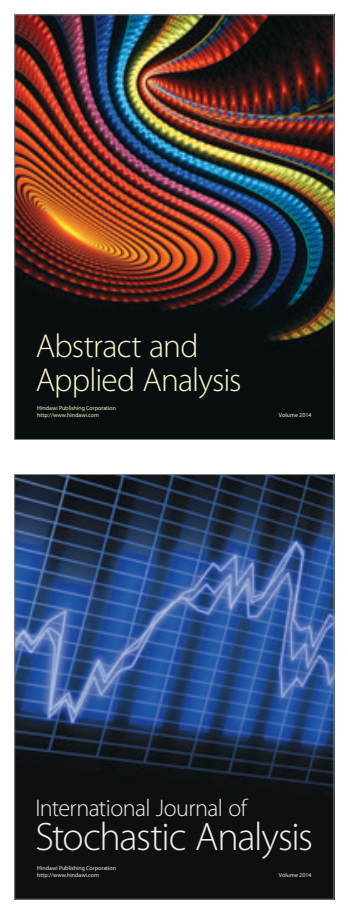

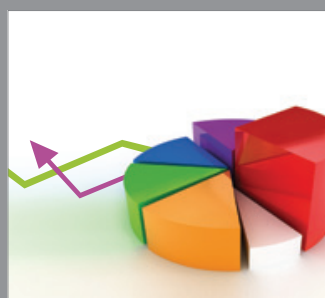

ournal of

Probability and Statistics

Promensencen
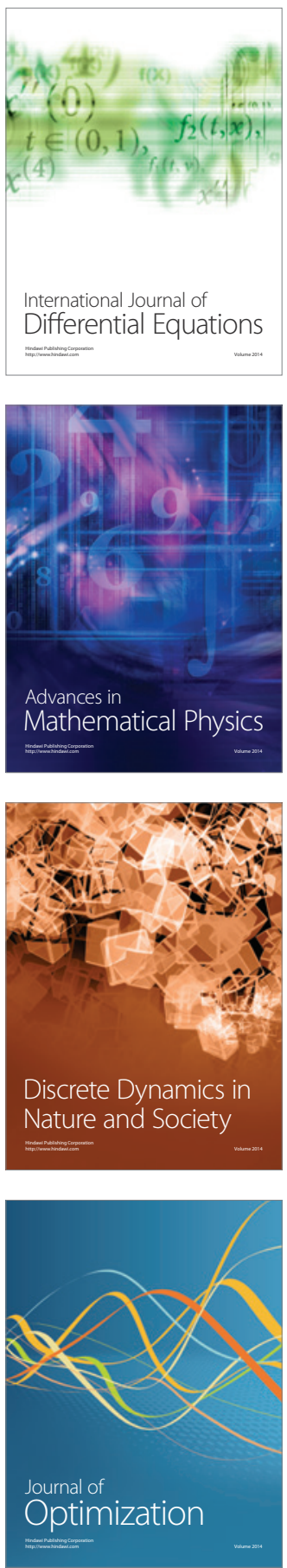